\title{
AVALIAÇÃO DA ESL-PBT NA DETERMINAÇÃO DE CARBARIL EM AMOSTRAS DE ABACAXI PÉROLA (ANANÁS COMOSUS) COMERCIALIZADAS NO MUNICÍPIO DE ITUMBIARA.
}

\author{
Roberta Correia Neto Nunes ${ }^{1}$, Allyson Leandro Rodrigues dos Santos ${ }^{2}$, Rogério Pacheco Rodrigues ${ }^{1}$, Alessandra Timóteo Cardoso ${ }^{1}$, \\ Adilson Correia Goulart ${ }^{1}$, João Paulo Victorino Santos ${ }^{3}$, Simone Machado Goulart ${ }^{3}$ \\ ${ }_{1}^{1}$ Laboratório de Química Analítica, Instituto Federal de Goiás - Campus Itumbiara, 75.524-010, Itumbiara-GO, Brasil. \\ ${ }^{2}$ Departamento de Química, Universidade Federal de Uberlândia-Campus Pontal, 38304-402, Ituiutaba-MG, Brasil. \\ 3 Departamento de Áreas Acadêmicas, Instituto Federal de Goiás - Campus Itumbiara, 75.524-010, Itumbiara-GO, Brasil.
}

*E-mail: simonemgoulart@yahoo.com.br

\section{RESUMO}

O uso de agrotóxicos no Brasil tem impulsionado várias pesquisas em análises de alimentos, visto que, o país está em posição de destaque no ranking dos maiores consumidores destes compostos. A Agência Nacional de Vigilância Sanitária (ANVISA), por meio do Programa de Análises de Resíduos de Agrotóxicos em Alimentos (PARA) realiza o monitoramento da contaminação dos alimentos; observando, desta forma, se os produtores estão fazendo uso correto destes compostos. Diante disto, o objetivo desta pesquisa foi otimizar o método de ESL-PBT para determinação de carbaril em amostras de abacaxi Pérola. O abacaxi foi escolhido devido à grande comercialização na região de Itumbiara-GO e por ter sido uma das frutas que apresentou resíduos de agrotóxicos acima do permitido no relatório PARA, nos anos de 2013 a 2015, divulgado em 2016. Para otimização do método ESL-PBT foi empregado um planejamento fatorial completo $2^{3}$, para avaliação do comportamento simultâneo de três fatores (relação do volume de água e solvente extrator, adição de $\mathrm{NaCl}$ e solução extratora) em triplicata. Para separação, identificação e quantificação de carbaril foi utilizada a cromatografia líquida de alta eficiência com detector ultravioleta/visível. O método ESL-PBT aplicado na determinação de carbaril em amostras de abacaxi mostrou-se simples e eficaz, empregando pequenas quantidades de solventes e amostras, com boa porcentagem média de recuperação. A porcentagem de recuperação do carbaril em abacaxi foi de 103,10\% e os experimentos com amostras reais de abacaxi, comercializados no município de Itumbiara-GO, não apresentaram a presença de carbaril ou esses estavam abaixo do limite de detecção do método.

Palavras-chave: Carbaril. Abacaxi. ESL-PBT.

\section{Introdução}

A Lei $\mathrm{n}^{\circ} 19.423$ de 26 de julho de 2016 , considera que agrotóxicos são produtos e agentes de processos físicos, químicos e biológicos, destinados à utilização nos setores de produção, no armazenamento e beneficiamento de produtos agrícolas, nas pastagens, na proteção de florestas nativas ou implantadas em outros ecossistemas, ambientes urbanos, hídricos e industriais; cuja finalidade seja alterar a composição da flora e da fauna a fim de preservá-las da ação danosa de seres vivos considerados nocivos [1].

Segundo dados do Sindicato Nacional da Indústria de Produtos para Defesa Agrícola (SINDAG), a indústria química produtora de agrotóxicos faturou, no Brasil, em 2011, 8,5 bilhões de dólares com tais produtos. $\mathrm{E}$, ao contrário da argumentação de que os problemas com o uso desses defensivos ocorrem mais entre os pequenos agricultores, os dados mostram que os maiores usuários de agrotóxicos são os grandes proprietários de terras. Conforme dados do IBGE e do SINDAG, das propriedades rurais de 0 a 10 hectares, 27\% usam agrotóxicos; em contrapartida, das de 10 a 100 hectares, $36 \%$ usam e das propriedades maiores que 100 hectares, $80 \%$ usam agrotóxicos [2].

$\mathrm{O}$ carbaril (1-naftil $\mathrm{N}$-metilcarbamato), é um inseticida da classe dos carbamatos, considerado altamente tóxico (classe toxicológica II); fórmula $\mathrm{C}_{12} \mathrm{H}_{11} \mathrm{NO}_{2}$ e com aplicação foliar nas culturas de abacaxi, abóbora, algodão, alho, banana, batata, cebola, couve-flor, feijão, maçã, pepino, repolho e tomate. A Figura 1 apresenta a fórmula estrutural do carbaril [3].

No Brasil, com o objetivo de avaliar continuamente os níveis de resíduos de agrotóxicos nos alimentos in natura que chegam à mesa do consumidor, foi criado em 2001 o Programa de Análises de Resíduos de Agrotóxicos (PARA) coordenado 
pela Agência Nacional de Vigilância Sanitária (ANVISA), contribuindo para a segurança alimentar, orientando as cadeias produtivas sobre as inconformidades existentes em processos produtivos, incentivando a adoção das Boas Práticas Agrícolas (BPA) e dando capacidade ao Governo de atender a segurança alimentar, evitando possíveis danos à saúde da população [4].

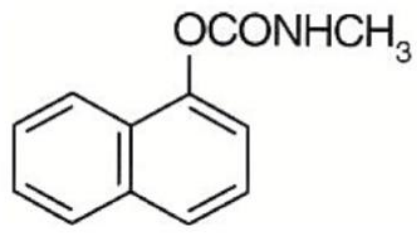

Figura 1 - Estrutura química do carbaril [3]

Com base nos resultados do relatório de pesquisas desenvolvidas pelo PARA nos anos de 2013 a 2015, divulgado em 2016, foram analisadas 240 amostras de abacaxi. Destas, 203 amostras foram consideradas satisfatórias, 129 não apresentaram resíduos dos agrotóxicos pesquisados e 74 apresentaram concentrações iguais ou inferiores ao limite máximo de resíduo (LMR) permitido [4].

O abacaxi tem cultivo mundial e no Brasil a espécie Ananas comusus, variedade Pérola, é a de maior importância econômica, sendo responsável por $88 \%$ da produção nacional e por quase $100 \%$ da produção comercial no Nordeste e Norte do País. [5].

A fruta é considerada a quarta mais cultivada no país, tendo importante papel social, por gerar emprego e renda no meio rural [6]. As pragas mais comuns que atacam as culturas são a broca do fruto (Thecla basalides) e a cochonilha (Dysmicoccus brevipes). A broca do fruto é a larva de uma pequena borboleta que ataca a inflorescência do abacaxi, cavando galerias e provocando o aparecimento de uma substância com aspecto de goma. O tratamento é feito com carbaril (260 gramas em 100 litros d'água), na base de 30 a 50 $\mathrm{mL}$ da solução por planta, tem aplicação foliar com LMR de $0,5 \mathrm{mg} \mathrm{kg}^{-1}$ sendo necessário um intervalo de segurança de sete dias [5].

Assim, inúmeras metodologias têm sido desenvolvidas para análise de resíduos de agrotóxicos em alimentos, utilizando a técnica de cromatografia líquida de alto eficiência (CLAE). O método analítico utilizado pela ANVISA para extração de carbaril em abacaxi, é o QuEChERS (Quick, Easy, Cheap, Effective, Rugged, Safe) [7-9]. Este já foi aplicado em diversas amostras de alimentos e bebidas, como: Cebola [10], soja [11], banana, mamão e pimentão [12], maçã, morango e tomate [13], laranja, [14], pera, pepino e repolho [15], mel [16], leite bovino [17], sucos de uva [18] e caldo de cana [19].

Em contrapartida, outro método que foi desenvolvido e que tem sido utilizado com sucesso para determinação de agrotóxicos em alimentos e demais amostras por cromatografia é a extração sólido-líquido com partição em baixa temperatura (ESL/PBT) [20-21]. O método permite o desempenho simultâneo pela extração de agrotóxicos e limpeza dos extratos e inúmeros estudos também já foram realizados, sendo aplicado em amostras frutíferas e de origem vegetal, como na batata [22], cenoura [23], abobrinha [24], alface [25], alho [26], milho [27], tomates [28], cajá-manga [29], pimentão [30] e aplicado em amostras de solos [31-33]. O método é simples, eficiente, oferece praticidade, baixo consumo de solvente orgânico e amostra, número reduzido de etapas e confiabilidade com porcentagens de recuperação elevadas e boa repetitividade [34].

O objetivo deste estudo foi otimizar o método ESL/PBT para determinação de carbaril em abacaxi por cromatografia líquida de alta eficiência com detector ultravioleta/visível (UVVis) e verificar a presença deste composto em amostras de abacaxi comercializadas no município de Itumbiara-Goiás, Brasil.

\section{Parte experimental}

A aplicabilidade e a otimização do ensaio experimental consistiram na utilização da técnica ESL-PBT, seguida de análise por HPLC-UV, conforme metodologia proposta por Morais et al. [35].

\subsection{Reagentes e soluções}

Foram utilizados acetato de etila $(99,5 \%$ v/v Mallinckrodt/HPLC), acetonitrila $(99,5 \% \quad \mathrm{v} / \mathrm{v} \quad-\quad$ J.T. Baker/HPLC) e cloreto de sódio (99,5\% m/m - Synth). Uma solução estoque de carbaril a $100,0 \mathrm{mg} \mathrm{L}^{-1}(99,8 \% \mathrm{~m} / \mathrm{m}-$ Sigma Aldrich) foi preparada em acetonitrila. A partir da solução estoque, foram preparadas soluções padrão de trabalho, contendo o carbaril em diferentes concentrações.

\subsection{Método ESL-PBT}

De início, empregou-se a metodologia de extração do carbaril no abacaxi utilizando o método de ESL-PBT, seguido de análise por CLAE-UV, conforme metodologia proposta por Morais et al. [35] com modificações.

Para o estudo, foram utilizadas amostras de abacaxi pérola cultivado na zona rural de Itumbiara-GO, Brasil, sem a utilização de agrotóxicos.

Após coleta, as amostras foram refrigeradas e analisadas no Laboratório de Análise Química Instrumental do Instituto Federal de Goiás - Campus Itumbiara (IFG-Itumbiara), onde foram trituradas conjuntamente, casca e polpa, em multiprocessador doméstico.

Posteriormente, a amostra foi dividida em duas partes onde uma parcela foi destinada a extração no branco da amostra e a outra fração para fortificação com princípio ativo de carbaril. Para o processo de fortificação, foi pesada $1,0 \mathrm{~g}$ de amostra em vial, com capacidade de $22 \mathrm{~mL}$ em material de vidro e tampa. 
Em seguida, as amostras foram fortificadas com 200,0 $\mu \mathrm{L}$ de solução preparada em acetonitrila ( $\mathrm{ACN}$ ) e padrão carbaril na concentração de $100 \mathrm{mg} \mathrm{L}^{-1}$, a fim de se obter, nos extratos finais, a concentração de $5 \mathrm{mg} \mathrm{L}^{-1}$. Após a fortificação, as amostras foram deixadas em repouso por um período de 3 horas para evaporação da $\mathrm{ACN}$ e, posteriormente, foram submetidas ao processo de ESL-PBT.

Para a extração do carbaril, foram adicionados ao sistema $2 \mathrm{~mL}$ de água deionizada, $4 \mathrm{~mL}$ de acetonitrila e agitação em vórtex por 60 segundos. Depois de realizar a etapa de extração, o sistema foi colocado no freezer, em temperatura de, aproximadamente, $-20{ }^{\circ} \mathrm{C}$ por um período de 3 horas para a partição das fases. Após este período, foi retirada uma alíquota do sobrenadante para análise em cromatógrafo a líquido de alta eficiência, acoplado a detector ultravioleta (CLAE-UV).

\subsection{Condições Cromatográficas}

As condições cromatográficas utilizadas foram: Detector de UV; Coluna Kinetex 5 um EVO C 18 - 150 X 4,6 mm; fase móvel: acetonitrila: água purificada $35: 65 \mathrm{v} / \mathrm{v}$, vazão da fase móvel: $0,8 \mathrm{~mL} \mathrm{~min}^{-1}$, temperatura da coluna: $35^{\circ} \mathrm{C}$ e volume de injeção $20 \mu \mathrm{L}$. Agitador de tubos vórtex (Biomixer, velocidade $2.800 \mathrm{rpm}$, potência $60 \mathrm{~W}$; Freezer vertical (Modelo CVU20GB Consul); Balança analítica de precisão com capela (Shimadzu ATX224, capacidade $220 \mathrm{~g}$ ); Cromatógrafo a líquido Shimadzu LC com detector UV-VIS $(\lambda: 213 \mathrm{~nm})$
Shimadzu SPD; sistema de operação LabSolutions Shimadzu. Para calcular as porcentagens de recuperação, foi construída uma curva analítica em acetonitrila, na faixa de concentração de $0,1 \mathrm{mg} \mathrm{L}^{-1}$ a $10 \mathrm{mg} \mathrm{L}^{-1}$.

\subsection{Otimização do método ESL-PBT e planejamento fatorial $2^{3}$ \\ Para otimização da ESL-PBT, empregou-se um} planejamento fatorial completo, $2^{3}$, para avaliação do comportamento simultâneo de três fatores: relação do volume de água e solvente extrator (1:1 ou 1:2), concentração salina (sem ou com $1,5 \%(\mathrm{~m} / \mathrm{v})$ de $\mathrm{NaCl}$ ) e tipo de solvente extrator (acetonitrila- ACN ou acetonitrila: acetato de etila - ACN/ACE $81,25: 18,75)$. O volume de solvente extrator foi fixado em 4 $\mathrm{mL}$.

Esses fatores são os mais citados na literatura como determinantes para uma melhor recuperação dos analitos [36]. $\mathrm{Na}$ Tabela 1, está apresentado o planejamento fatorial $2^{3}$ utilizado nas análises estatísticas para otimização do método. Para análise estatística, foi utilizado o teste de $t$-student ao nível de $95 \%$ de confiança e 5\% significância. As planilhas eletrônicas utilizadas para análise dos resultados foram as de Teófilo e Ferreira [37]; que possibilitaram uma interpretação rápida e eficiente na escolha das melhores condições na otimização do método ESL-PBT. Os três fatores serão estudados em dois níveis e as análises, realizadas em duplicata.

Tabela 1. Planejamento fatorial $2^{3}$, em triplicata, realizado para estabelecer melhores condições para extração de carbaril de amostras de abacaxi.

\section{Fatores Codificados}

\begin{tabular}{llccc} 
Ensaios & Replicatas & $F(1)$ & $F(2)$ & $F(3)$ \\
& & & & \\
\cline { 3 - 5 } 1 & 1,2 e 3 & - & - & - \\
2 & 4,5 e 6 & + & - & - \\
3 & 7,8 e 9 & - & + & - \\
4 & 10,11 e 12 & + & + & - \\
5 & 13,14 e 15 & - & - & + \\
6 & 16,17 e 18 & + & - & + \\
7 & 19,20 e 21 & - & + & + \\
8 & 22,23 e 24 & + & + & + \\
\hline
\end{tabular}

\section{Fatores Originais}

(1) Relação do

volume de água e solvente extrator
(2) Adição $\mathrm{NaCl}$ $(\% \mathrm{~m} / \mathrm{v})$
(3) Solução Extratora

\begin{tabular}{lccc}
- & $1: 1$ & 0 & ACN \\
- & $1: 2$ & 0 & ACN \\
- & $1: 1$ & 1,5 & ACN \\
- & $1: 2$ & 1,5 & ACN \\
+ & $1: 1$ & 0 & ACN/ACE \\
+ & $1: 2$ & 0 & ACN/ACE \\
+ & $1: 1$ & 1,5 & ACN/ACE \\
+ & $1: 2$ & 1,5 & ACN/ACE \\
\hline
\end{tabular}

\subsection{Limite de detecção e quantificação}

Os limites de detecção (LOD) e quantificação (LOQ) do método proposto foram determinados pela fortificação dos extratos de abacaxi (obtidos pelo método ESL-PBT) em concentrações decrescentes. LOD e LOQ foram considerados como três e dez vezes, respectivamente. Este método é conhecido como método do sinal-ruído.
2.6 Aplicação do método otimizado em amostras reais de abacaxi comercializados em Itumbiara-GO

Após a otimização do método, amostras de abacaxi foram coletadas em estabelecimentos comerciais de ItumbiaraGO. Sendo assim, o melhor resultado dos 8 ensaios do planejamento foi escolhido para a análise das amostras reais de abacaxi. As amostras de abacaxi pérola (Ananás comosus) foram coletadas em pontos diferentes, estes três tipos diferentes 
de comercialização foram selecionados: Supermercado (Amostra A), Feira Livre da cidade (Amostra B) e Vendedores ambulantes sem ponto comercial fixo (Amostra C).

\section{Resultados e discussão}

\subsection{Curva Analítica}

Para confecção da curva analítica foram realizadas injeções de soluções do padrão de carbaril, com o objetivo de verificar a resposta do detector frente às diferentes concentrações do analito, assim diluindo, foi obtido os padrões nas concentrações de $\left(0,5 \mathrm{mg} \mathrm{L}^{-1}, 1 \mathrm{mg} \mathrm{L}^{-1}, 2 \mathrm{mg} \mathrm{L}^{-1}, 5 \mathrm{mg} \mathrm{L}^{-1}\right.$ e $10 \mathrm{mg} \mathrm{L}^{-1}$ ). Com as respostas obtidas pelo detector foi construída a curva analítica.

A equação da reta obtida na curva analítica foi $y=471718 x-45334$ e coeficiente de determinação de 0,9907 indicando a linearidade das respostas do detector na faixa de concentração estudada. Esse resultado está de acordo com o estabelecido no guia SANTE/11813/2017 [38].

\subsection{Análise do método ESL-PBT}

No sentido de otimizar a massa de amostra utilizada e volume de solvente extrator, foram realizadas modificações no método proposto por Morais et al. [35]. Os autores utilizaram 3,0 g de amostra de abacaxi, 8,5 $\mathrm{mL}$ de solvente extrator. No presente trabalho, selecionou-se para 1,0 g de amostra, $4 \mathrm{~mL}$ de solvente extrator. Na Tabela 2 são apresentadas as porcentagens de recuperação (\%R), assim como a média e seu respectivo coeficiente de variação $(\mathrm{CV})$.

\begin{tabular}{cccc}
\multicolumn{4}{l}{ Tabela 2. Porcentagem de recuperação do carbaril em amostras de abacaxi } \\
\hline Replicatas & $\% \mathbf{R}$ & Média (\%) & CV (\%) \\
\hline $\mathbf{1}$ & $94 \%$ & & \\
$\mathbf{2}$ & $114 \%$ & $106 \%$ & $9,90 \%$ \\
$\mathbf{3}$ & $110 \%$ & & \\
\hline
\end{tabular}

Analisando a Tabela 2, é possível inferir a eficiência da ESL- PBT na determinação do carbaril em abacaxi, uma vez que as porcentagens de recuperação ficaram dentro da faixa de aceitação de 70 a $120 \%$ de recuperação e coeficiente de variação bem abaixo do nível aceitável de $20 \%$ conforme estabelecido no guia SANTE / 11813/2017 [38] e recomendado por Ribani et al. [39].

Goulart et al. [40] também comprovaram a eficiência deste método ESL-PBT na recuperação de carbofurano em amostras de solo, obtendo médias de recuperação de 70 a $85 \%$.

\subsection{Seletividade do método}

$\mathrm{Na}$ Figura 2, estão apresentados os cromatogramas resultantes da ESL-PBT em amostras de abacaxi sem o agrotóxico (branco da amostra), comparadas com padrão de carbaril a $4 \mathrm{mg} \mathrm{L}^{-1}$.

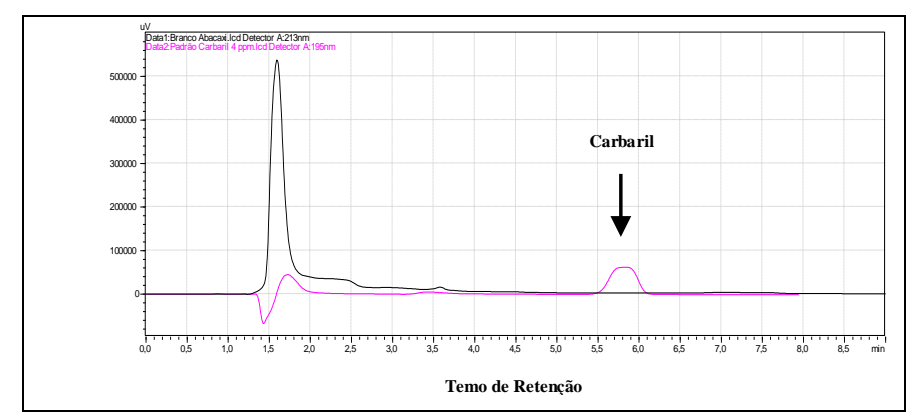

Figura 2. Cromatograma do branco da amostra em comparativo com uma solução do padrão carbaril a $4 \mathrm{mg} \mathrm{L}^{-1}$.

Observa-se que o carbaril teve um tempo de retenção igual a 5,8 minutos e ausência de interferentes no tempo de retenção do princípio ativo, demonstrando a seletividade do método.

\subsection{Avaliação do método ESL-PBT com utilização do planejamento fatorial $2^{3}$}

Para o processo de otimização do método e análise do carbaril em abacaxi, foi conduzido um planejamento fatorial, no qual trabalhou-se três efeitos principais: (1) relação do volume de água e solvente extrator, (2) adição de $\mathrm{NaCl}$, (3) solução extratora, com três interações de dois efeitos, 12,13 e 23 e uma interação de três efeitos, 123. A Tabela 3 apresenta as porcentagens médias de recuperação, os efeitos principais referentes a cada fator e ainda a interação entre eles.

$\mathrm{Na}$ Figura 3, nota-se que o aumento do volume de solvente extrator, de 2 para $4 \mathrm{~mL}$, reduziu as porcentagens de recuperação do carbaril na amostra abacaxi em 5,04\%. Ao utilizar a mistura dos solventes, saindo do nível mais baixo para o nível mais alto, a redução na porcentagem de recuperação do carbaril é ainda mais expressiva 27,68\%.

Avaliando o comportamento do fator (3) é perceptível que, partindo do nível mais baixo (solução extratora $\mathrm{ACN}$ ) para o nível mais alto (solução extratora ACN/ACE), temos um aumento de $2,56 \%$ na recuperação média. No entanto, este comportamento não se repete quando se utiliza $4 \mathrm{~mL}$ de solução extratora; nesta condição, acontece uma redução de 20,08\% na recuperação média do carbaril na amostra estudada. Desta forma, verifica-se que a maior porcentagem média de extração do carbaril na amostra abacaxi foi obtida utilizando 2 $\mathrm{mL}$ de água e $2 \mathrm{~mL}$ da solução extratora ACN/ACE. 


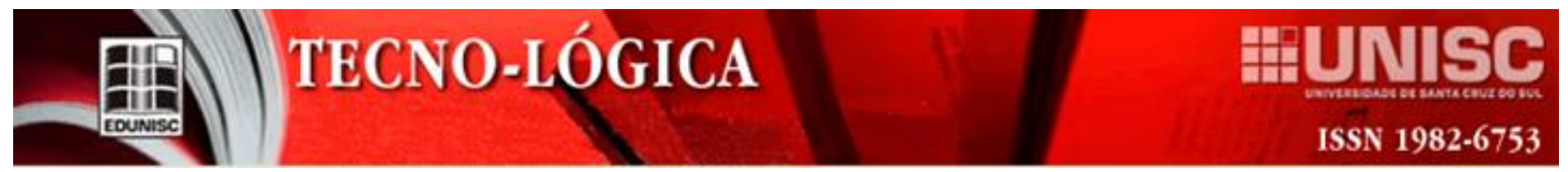

Vieira et al. [41] obteve melhores resultados quando na adição de acetonitrila e acetato de etila, visto que esse é menos polar que a acetonitrila, e como os piretróides são hidrofóbicos, a adição de acetato de etila favoreceu na extração. Esses resultados foram parecidos com os encontrados neste trabalho, o carbaril é um composto formado por um anel benzênico, hidrofóbico, apresentando melhores resultados quando se utiliza a mistura extratora acetonitrila e acetato de etila (ACN/ACE).

Tabela 3: Porcentagem de recuperação média (\%R.M), efeitos principais e interação dos fatores referente ao planejamento fatorial $2^{3}$.

\begin{tabular}{|c|c|c|c|c|c|c|c|c|c|}
\hline Ensaios & Fator (1) & Fator (2) & Fator (3) & $\%$ R.M & & Efeitos & & Erros & p-valor \\
\hline 1 & 01:01 & 0 & $\mathrm{ACN}$ & 97,03 & Fator 1* & $-16,44$ & \pm & 4,35 & $\mathbf{0 , 0 1}$ \\
\hline 2 & 01:02 & 0 & $\mathrm{ACN}$ & 97,375 & Fator 2 & 4,42 & \pm & 4,35 & 0,34 \\
\hline 3 & 01:01 & 1,5 & $\mathrm{ACN}$ & 103,35 & Fator 3 & $-8,67$ & \pm & 4,35 & 0,08 \\
\hline 4 & 01:02 & 1,5 & $\mathrm{ACN}$ & 92,95 & Interação 1x2 & 1,43 & \pm & 4,35 & 0,75 \\
\hline 5 & 01:01 & 0 & ACN/ACE & 103,1 & Interação1x3* & $-11,41$ & \pm & 4,35 & $\mathbf{0 , 0 3}$ \\
\hline 6 & 01:02 & 0 & $\mathrm{ACN} / \mathrm{ACE}$ & 67,02 & Interação 2x3 & 3,47 & \pm & 4,35 & 0,45 \\
\hline 7 & 01:01 & 1,5 & ACN/ACE & 102,76 & Interação 1x2x3 & 6,80 & \pm & 4,35 & 0,16 \\
\hline 8 & 01:02 & 1,5 & $\mathrm{ACN} / \mathrm{ACE}$ & 83,14 & & & & & \\
\hline
\end{tabular}

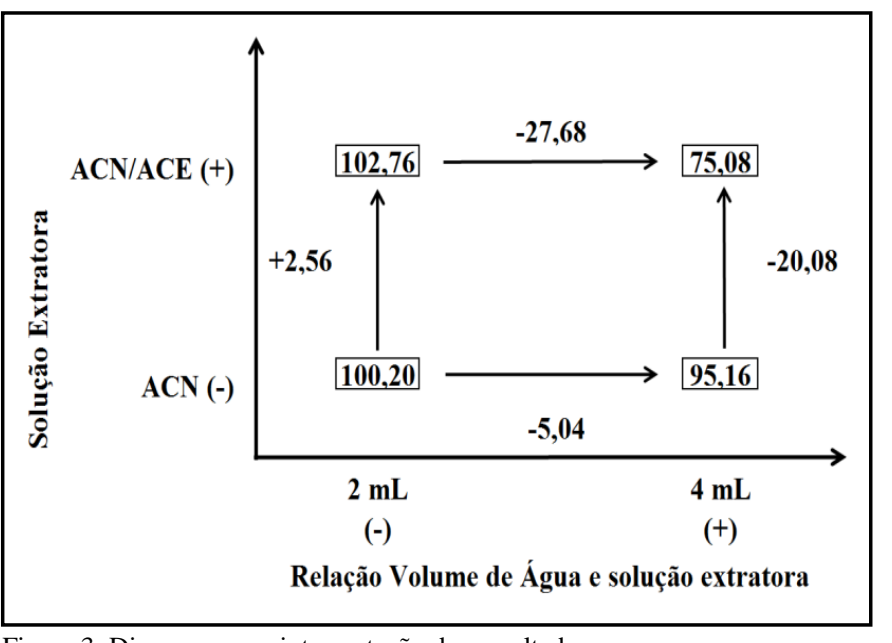

Figura 3. Diagrama para interpretação dos resultados.

Deste modo, levando em consideração a análise estatística apresentada na Tabela 3 e ainda o estudo realizado com a ajuda do diagrama da Figura 3, o ensaio 5 foi escolhido para as análises das amostras reais de abacaxi. Como a adição de sal não foi estatisticamente significativa na extração do carbaril, optou-se por não acrescentar a etapa de adição de sal ao sistema, o que tornaria a análise mais demorada e com maior custo.

Assim sendo, o método otimizado para a determinação e quantificação do princípio ativo carbaril em amostras de abacaxi consiste em pesar 1,0 grama de abacaxi triturado, polpa e casca, seguindo da adição de $2 \mathrm{~mL}$ de água e $2 \mathrm{~mL}$ da mistura extratora ACN/ACE na proporção 81,25:18,75, v/v; em seguida, agitar o sistema em agitador do tipo vortex por 60 segundos. Como visto, o método otimizado demonstrou ser rápido e com poucas etapas. $\mathrm{O}$ uso de $2 \mathrm{~mL}$ de solução extratora além de diminuir os custos de análise, também promove uma maior concentração do extrato, o que permite limites de quantificação menores nas amostras reais. Os limites de detecção e quantificação do método proposto, determinados pelo método do sinal-ruído foram $0,04 \mathrm{mg} \mathrm{kg}^{-1}$ e $0,13 \mathrm{mg} \mathrm{kg}^{-1}$, respectivamente.

\subsection{Aplicação em amostras comerciais}

Para análise das amostras reais de abacaxi, foram adquiridos frutos comercializados no município de ItumbiaraGO. As amostras foram submetidas ao método ESL-PBT otimizado, como descrito no ensaio 5 da Tabela 1.

$\mathrm{Na}$ Figura 4, são apresentados os cromatogramas resultantes do processo de extração das amostras reais em comparativo com um extrato do padrão carbaril na concentração de $4 \mathrm{mg} \mathrm{L}^{-1}$.

Como pode ser observado na Figura 4, a linha correspondente à solução do padrão carbaril (-) apresentou um pico aos 5,8 minutos, indicando o tempo de retenção do carbaril. Observa-se, também, que em nenhum dos três pontos de coleta foi observado pico cromatográfico no tempo de retenção do carbaril. Desta forma, as amostras comercializadas nos pontos de coleta escolhidos, possivelmente não estão contaminadas com o princípio ativo carbaril ou então os resíduos deste agrotóxico nos frutos estão abaixo do limite de deteç̧ão e quantificação do método. O limite de detecção do carbaril no equipamento utilizado é de $0,1 \mathrm{mg} \mathrm{L}^{-1}$, ou seja, essa foi a menor concentração do agrotóxico capaz de gerar um sinal no detector igual a três vezes o ruído do equipamento. Os limites de detecção e quantificação do método, também determinados pelo método do sinal-ruído, foram $0,04 \mathrm{mg} \mathrm{kg}_{-1}$ e 0,13 $\mathrm{mg} \mathrm{kg}-1$, respectivamente. Resultados semelhantes foram obtidos por Rodrigues e colaboradores na análise de carbofurano em Cucurbita pepo l ("abobrinha") [24]. 


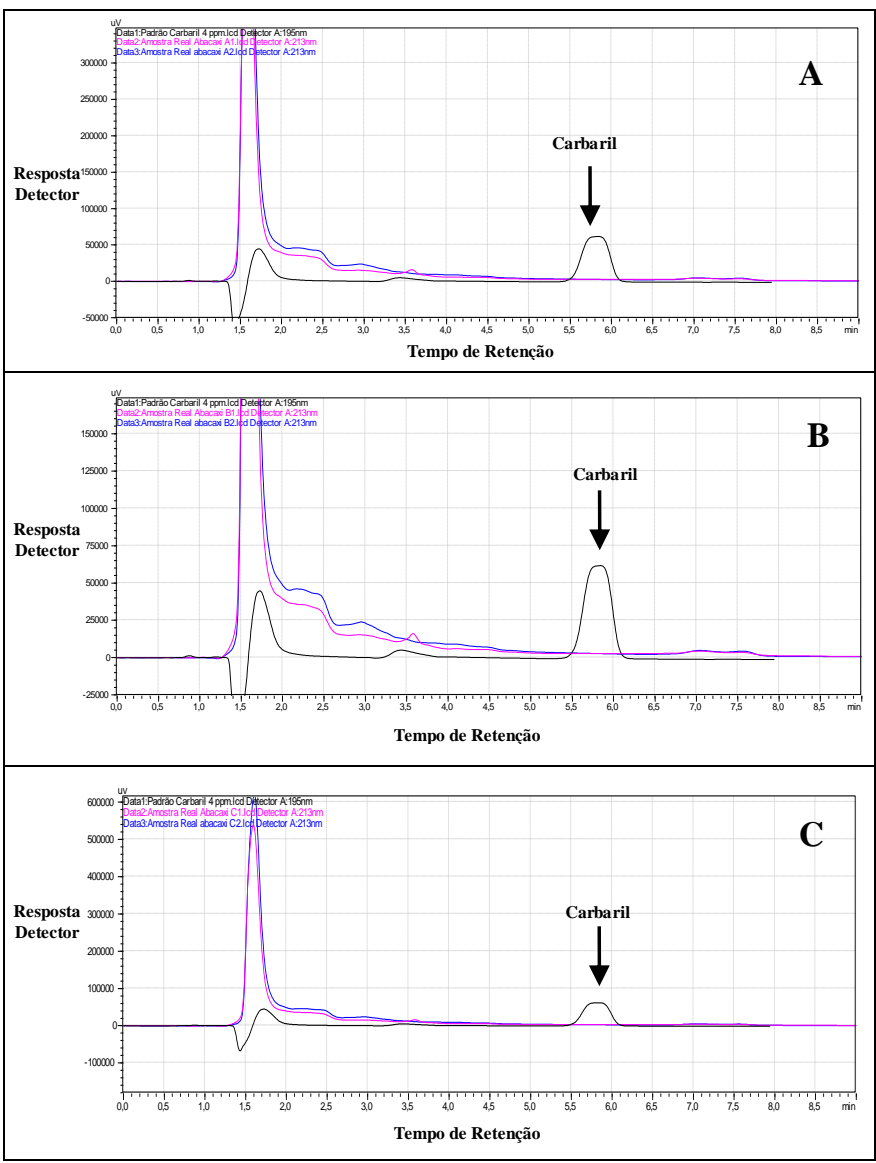

Figura 4. Comparativo entre o extrato de uma solução do padrão carbaril a 4 ppm com (A) Amostras de abacaxi coletadas em supermercado, (B) Feira Livre, (C) Vendedores Ambulantes.

\section{Conclusão}

O método otimizado para a determinação e quantificação do carbaril em amostra de abacaxi, utilizando a técnica de ESL-PBT e análise por cromatografia líquida de alta eficiência, mostrou-se eficiente e prático. Como visto, com poucas etapas, baixos volumes de solvente e amostra, foi possível identificar e quantificar o princípio ativo estudado.

As análises realizadas nas amostras reais, coletadas no município de Itumbiara-GO, não apresentaram resíduos de carbaril em nenhum dos três tipos de comercialização. Desta forma, conclui-se que os frutos coletados para este estudo, possivelmente não estavam contaminados com carbaril ou o princípio ativo estava abaixo do limite de detecção do método.

\section{Agradecimentos}

Ao Ministério da Educação (MEC) e a Fundação Nacional de Desenvolvimento da Educação (FNDE) pelo o apoio financeiro concedido ao Programa de Educação Tutorial
(PET) do Instituto Federal de Goiás - Campus Itumbiara e ao Núcleo de Pesquisa e Estudos em Química de Goiás (NUPEQUI).

\section{AVALIATION OF THE SLE-LTP IN THE DETERMINATION OF CARBARYL IN SAMPLES OF PEARL PINEAPLE (ANANAS COMOSUS) COMMERCIALIZED IN THE ITUMBIARA CITY}

ABSTRACT: The use of pesticides in Brazil has driven several researches in food analysis, since the country is in a position of prominence in the ranking of the largest consumers of these compounds. The National Agency of Sanitary Surveillance (ANVISA), through the Program of Analysis of Residues of Agrochemicals in Foods (PARA), monitors the contamination of food, observing in this way if the producers are making correct use of these compounds. Therefore, the objective of this research was to optimize the method Solid-Liquid Extraction with Low Temperature Partition (SLE-LTP) for determination of carbaryl in samples of Pineapple Pearl. The pineapple was chosen because of the great commercialization in the region of Itumbiara-GO and because it was one of the fruits that presented pesticide residues above the allowed in the report PARA in the years 2013 to 2015, released in 2016. For optimization of the SLE-LTP method a complete factorial design $2^{3}$ was used to evaluate the simultaneous behavior of three factors (volume ratio of water and solvent extraction, addition of $\mathrm{NaCl}$ and extraction solution) in triplicate. For the separation, identification and quantification of carbaryl, High Performance Liquid Chromatography with Ultraviolet Detector (HPLC-UV) was used. The SLE-LTP method applied in the determination of carbaryl in pineapple samples was simple and efficient, consuming small amounts of solvents and samples, with good average recovery percentage. The percentage of recovery of carbaryl in pineapple was $103.10 \%$ and the experiments with real samples of pineapple marketed in the Itumbiara-GO did not present the presence of carbaryl or those were below the limit of detection of the analysis method.

Keywords: Carbaryl. Pineapple. SLE-LTP.

\section{Referências}

[1] GOIÁS. Decreto na Lei No 19.423 , de Julho de 2016. Produção, o armazenamento, o comércio, o transporte interno, a utilização, o destino final de resíduos e embalagens, a inspeção e a fiscalização de agrotóxicos, seus componentes e afins, no Estado de Goiás, e dá outras providências. Palácio do Governo do Estado de Goiás, em Goiânia, 26 de julho de 2016. Disponível em $<$ http://www.gabinetecivil.go.gov.br/pagina_leis.php?id=21279> acessado em 27 de abril de 2019. 
[2] CONSEA. Conselho Nacional de Segurança Alimentar e Nutricional. Relatório final sobre os impactos dos agrotóxicos na soberania e segurança alimentar e nutricional e no direito humano à alimentação adequada. (2014) Disponível em < http://www4.planalto.gov.br/consea/publicacoes/segurancaalimentar-e-nutricional/a-seguranca-alimentar-e-nutricional-e-o-direitohumano-a-alimentacao-adequada-no-brasil-indicadores-emonitoramento/relatorio-consea.pdf > acessado em 12 de abril de 2019 .

[3] BRASIL. ANVISA. Agência Nacional de Vigilância Sanitária. Monografia do Carbaril. Brasília, 2006. Disponível em: <http://portal.anvisa.gov.br/documents/111215/117782/C03\%2B\%2BCarbaril. pdf/f2b52d86-280c-4899-8ec2-7ba6b929433f> acessado em 20 de abril de 2019.

[4] BRASIL. ANVISA. Agência Nacional de Vigilância Sanitária. Programa de Análise de resíduos de agrotóxicos em alimentos (PARA), 2016. Disponível em:<http://portal.anvisa.gov.br/documents/111215/0/Relat\%C3\%B3rio+PARA +2013-2015_VERS\%C3\%83O-FINAL.pdf/494cd7c5-5408-4e6a-b0e55098 cbf759f8> acessado em 01 de março de 2019.

[5] EMBRAPA. Empresa Brasileira de Pesquisa Agropecuária. Abacaxi. Disponível em: <https://www.embrapa.br/mandioca-efruticultura/cultivos/abacaxi> acessado em 14 de março de 2019.

[6] BRASIL. Ministério da Agricultura, Pecuária e Abastecimento. Produção integrada no Brasil: agropecuários sustentáveis alimentos seguros. $1^{\circ} \mathrm{ed}$. Secretária de Desenvolvimento Agropecuário e Cooperativismo. Brasília: Mapa/ACS, 2009

[7] PRESTES, O. D.; FRIGGI, C. A.; ADAIME, M. B.; ZANELLA, R. Um Método Moderno de Preparo de Amostra para Determinação Multirresíduo de Pesticidas em Alimentos por Métodos de Cromatográficos Acoplados à Espectrometria de Massas. Química Nova, v. 32, n. 6, p. 1620-1634, 2009.

[8] PRESTES, O. D.; ADAIME, M. B.; ZANELLA, R. QuEChERS: Possibilidades e Tendências no Preparo de Amostra para Determinação Multirresíduo de Pesticidas em Alimento. Scientia Chromatographia, v. 03, n. 01, p. 61-64, 2011

[9] QUEIROZ, S. C. N.; FERRACINI, V. L.; ROSA, M. A. Validação de Método Multirresíduo para Determinação de Pesticidas em Alimentos Empregando QuEChERS e UPLC-MS/MS. Química Nova, v. 35, n. 01, p. 185192, 2012.

[10] RODRIGUES, S. A.; CALDAS, S. S.; FURLONG, E. B.; PRIMEL, E. G. Otimização e Validação de Método Empregando QuEChERS Modificado e LC-ESI-MS/MS para Determinação de Agrotóxico em Cebola. Química Nova, v. 34, n. 05, p. 780-786, 2011.

[11] GOUVÊA, A. V.; CARDOSO, M. H. W. M.; BASTOS, L. H. P.; SILVA, C. B.; ORTIZ, N. D.; NÓBREGA, A. W.; JACOB, S. C. Validação de metodologia QuEChERS-acetato para a análise de multirresíduo de agrotóxicos em amostras de soja e de extrato solúvel de soja utilizando cromatografia líquida de alta eficiência acoplado à espectrometria de massas sequencial, Revista Instituto Adolfo Lutz, v. 73, n. 1, p. 40-58, 2014

[12] LEMOS, M. F.; LEMOS, M. F.; PACHECO, H. P.; SCHERER, R. Monitoring of organophosphorous pesticide residues in sample of Banana, Papaya, and Bell Pepper. Química Nova, v. 38, n. 2, p. 268-273, 2015

[13] LORENZ, J. G.; COSTA, L. L. F.; SUCHARA, E. A.; SANT’ANNA, E. S. Multivariate Optimization of the QuEChERS-GC-ECD Method and Pesticide Investigation Residues in Apples, Strawberries, and Tomatoes Produced in Brazilian South. Journal of the Brazilian Chemical Society, v. 25, n. 9 , p. $1583-1591,2014$.

[14] GOLGE, O.; KABAK, B. Determination of 115 pesticide residues in oranges by high-performance liquid chromatography-triple-quadrupole mass spectrometry in combination with QuEChERS method. Journal of Food Composition and Analysis, v. 41, p. 86-97, 2015.

[15] CHENG, Z.; DONG, F.; XU, J.; LIU, X.; WU, X.; CHEN, Z.; PAN, X ; GAN, J.; ZHENG, Y. Simultaneous determination of organophosphorus pesticides in fruits and vegetables using atmospheric pressure gas chromatography quadrupole-time-of-flight mass spectrometry. Food Chemistry, v. 231 , p. $365-373,2017$

[16] SAMPAIO, M. R. F.; TOMASINI, D.; CARDOSO, L. V.; CALDAS, S. S.; PRIMEL, E. G. Determination of Pesticide Residues in Sugarcane Honey by QuEChERS and Liquid Chromatography. Journal of the Brazilian Chemical Society, v. 23, n. 2, p. 197-205, 2012.

[17] HERNANDES, T.; DORES, E. F. G. C.; RIBEIRO, M. L.; ROSSIGNOLI, P. A.; MALM, O. Simple Mathod to Determine Residual Cypermethrin and Deltamethrin in Bovine Milk. Journal of the Brazilian Chemical Society, v. 25, n. 9 , p. $1656-1661,2014$

[18] SOUZA, D. F.; SOUZA, E. L.; BORGES, E. M. Determination of Pesticides in Grape Juices by QuEChERS and Liquid Chromatography-Tandem Mass Spectrometry. Journal of the Brazilian Chemical Society, v. 27, n. 9, p. 1626-1635, 2016.

[19] SUGANTHI, A.; Bhuvaneswari, K.; Ramya, M. Determination of neonicotinoid insecticide residues in sugarcane juice using LCMSMS. Food Chemistry, v. 241, p. 275-280, 2018

[20] GOULART, S. M.; QUEIROZ, M. E. L. R.; NEVES, A. A.; QUEIROZ, J. H. Low-temperature clean-up method for the determination of pyrethroids in milk using gas chromatography with electron capture detection. Talanta, v. 75 , n. 5 , p. $1320-1323,2008$

[21] PINHO, G. P.; NEVES, A. A.; QUEIROZ, M. E. L. R.; SILVÉRIO, F. O. Pesticide determination in tomatoes by solid-liquid extraction with purification at low temperature and gas chromatography. Food Chemistry, v. 121, n. 1, p. 251-256, 2010

[22] RIGUEIRA, L. M. B.; RIBEIRO, K. L.; QUEIROZ, M. E. L. R.; NEVES, A. A.; ZAMBOLIM, L.; OLIVEIRA, R. M. Determination of Chlorpyrifos and Thiamethoxam in Potato Tuber (Solanum tuberosum L.) and Soil of Brazil Using Solid-Liquid Extraction with Low Temperature Partitioning (SLE/LTP). Journal of the Brazilian Chemical Society, v. 24, n. 12, p. 2042-2049, 2013.

[23] ARAÚJO, E. A.; LARA, M. C. R.; REIS, M. R.; VIRIATO, R. L. S.; ROCHA, R. A. R.; GONÇALVES, R. G. L.; HELENO, F. F.; QUEIROZ, M E. L. R.; TRONTO, J.; PINTO, F. G. Determination of Haloxyfop-Methyl, Linuron, and Procymidone Pesticides in Carrot Using SLE-LTP Extraction and GC-MS. Food analytical methods, v.9, n. 5, p. 1344-1352, 2016.

[24] RODRIGUES, R. P.; FARIAS, W. R.; GOULART, S. M.; GOULART, A C.; SANTOS, J. P. V.; QUEIROZ, M. E. L. R. Otimização da extração sólidolíquido com partição em baixa temperatura para determinação de carbofurano em Cucurbita pepo 1 ("abobrinha") por cromatografia líquida de alta eficiência. Química Nova, v. 41, n. 2, p. 213-218, 2018

[25] COSTA, A. I. G.; QUEIROZ, M. E. L. R.; NEVES, A. A.; SOUSA, F. A.; ZAMBOLIM, L. Determination of pesticides in lettuce using solid-liquid extraction with low temperature partitioning. Food Chemistry, v. 181, p. 64-71, 2015

[26] MORENO, C. M.; STADLER, T.; SILVA, A. A.; BARBOSA, L. C. A. QUEIROZ, M. E. L. R. Determination of maleic hydrazide residues in garlic bulbs by HPLC. Talanta, v. 89, p. 369-376, 2012.

[27] FREITAS, R. S.; QUEIROZ, M. E. L. R.; FARONI, L. R. A.; HELENO, F. F.; MOURA, V. V. Desenvolvimento do Método de Extração SólidoLíquido com Partição em Baixa Temperatura para Determinação de Inseticidas em Grãos de Milho Ozonizados. Química Nova, v. 37, n. 2, p. 238-243, 2014. 


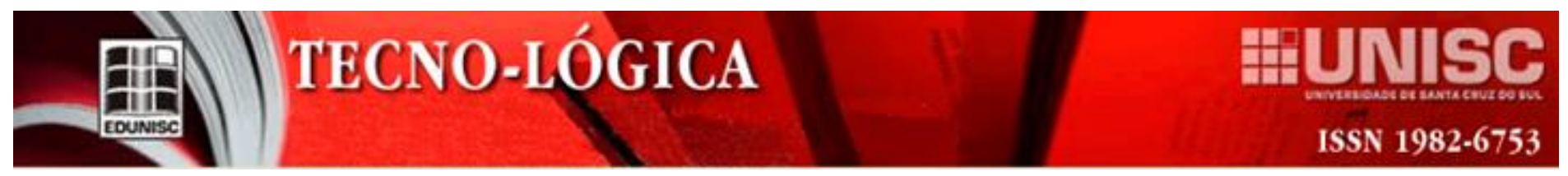

[28] PINHO, G. P.; NEVES, A. A.; QUEIROZ, M. E. L. R.; SILVÉRIO, F. O.; Pesticide determination in tomatoes by solid-liquid extraction with purification at low temperature and gas chromatography. Food Chemistry, v. 121, n. 1, p. 251-256, 2010.

[29] SANTANA, E. T. D.; SOARES, D. F.; FARIA, A. M. Development of a Methodology for the Determination of Pesticide Residues in Cajá-Manga Pulp (Spondias dulcis L.) Using Solid-Liquid Extraction with Low-Temperature Partitioning. Journal of Chemistry, v. 2018, p. 1-10, 2018 https://doi.org/10.1155/2018/6012503

[30] HELENO, F. F.; RODRIGUES, A. A. Z.; QUEIROZ, M. E. L. R.; NEVES, A. A.; OLIVEIRA, A. F.; LIBARDI, V. M. Determination of fungicides in bell pepper using solid-liquid extraction with low temperature partitioning. Microchemical Journal, 2019. Disponível em: <https://doi.org/10.1016/j.microc.2019.04.045> acessado em 24 de abril de 2019.

[31] ASSIS, E. C.; SILVA, A. A.; BARBOSA, L. C.; QUEIROZ, M. E. L. R.; D'ANTÔNIO, L.; GONÇALVES, V. A. Optimization and validation of the solid-liquid extraction technique for determination of picloram in soils by high Performance liquid chromatography. Planta Daninha, v. 29, n. 3, p. 683-696, 2011.

[32] SILVA, C. F.; BERNARDES, J. T.; GOULART, A. C.; GOULART, S. M. Estudo da Lixiviação do carbofurano em diferentes perfis de coluna de latossolo vermelho. Acta Brasiliensis, v. 2 n. 3, p. 96-99, 2018.

[33] GOULART, A. C.; GOULART, S. M.; MEDEIROS, M. S.; SANTOS, J. P. V. Avaliação da contaminação ambiental por carbofurano em solo proveniente do cultivo de cana-de-çúcar. Tecno-lógica, v. 22, n. 2, p. 187-193, 2018.

[34] HELENO, F. F.; QUEIROZ, M. E. L. R.; NEVES, A. A.; OLIVEIRA, A. F. Otimização, Validação e Aplicação de Método para Determinação da Concentração Residual de Difenoconazol em Morangos após Múltiplas Aplicações. Química Nova, v. 37, n. 1, p. 153-157, 2014.

[35] MORAIS, E. H. C.; RODRIGUES, A. A. Z.; QUEIROZ, M. E. L. R.; NEVES, A. A.; MORAIS, P. H. D. Determination of thiamethoxam, triadimenol and deltamethrin in pineapple using SLE-LTP extraction and gas chromatography. Food Control, v. 42, p. 9-17, 2014.

[36] GOUlarT, S. M.; ALVES, R. D.; NEVES, A. A.; QUEIROZ, J. H.; ASSIS, T. C.; QUEIROZ, M. E. L. R. Optimization and validation of liquidliquid extraction with low temperature partitioning for determination of carbamates in water. Analytica Chimica Acta, v. 671, n.1-2, p. 41-47, 2010.

[37] TEÓFILO, R. F.; FERREIRA, M. M. C. Quimiometria II: planilhas eletrônicas para cálculos de planejamentos experimentais, um tutorial. Química Nova, v. 29, n. 2, p. 338-350, 2006

[38] European Commission. SANTE/11813/2017. Disponível em: https://ec.europa.eu/food/sites/food/files/plant/docs/pesticides_mrl_guidelines_ wrkdoc_2017-11813.pdf, acessado em julho de 2019 .

[39] RIBANI, M.; BOTTOLI, C. B. G.; COLLINS, C H.; JARDIM, I. C. S. F.; MELO, L. F. C.; Validação em métodos cromatográficos e eletroforéticos. Química nova, v. 27, n. 5, p. 771-780, 2004.

[40] GOULART, A. C.; GOULART, S. M.; WENDLING, B.; MEDEIROS, M. S. Determinação de Carbofurano em Solo Utilizando a Extração SólidoLíquido e Partição a Baixa Temperatura. Tecnologia Educacional, v. 215, p. $16-23,2016$

[41] VIEIRA, H. P.; NEVES, A. A.; QUEIROZ, M. E. L. R. Otimização e Validação da Técnica de Extração Líquido-Líquido com Partição em Baixa Temperatura (ELL-PBT) para Piretróides em Água e Análise por CG. Química Nova, v. 30, p. 535-540, 2007.
[42] MARINHO, M. R. M.; CASTRO, W. B. Planejamento Fatorial: Uma ferramenta poderosa para os pesquisadores. In: XXX11l - Congresso Brasileiro de Engenharia, 2005, Anais...Campina Grande-PB, p.1-9, 2005. 